This manuscript is published and available in Death Studies $19^{\text {th }}$ March 2020 http://dx.doi.org/10.1080/07481187.2020.1739780

\title{
Grief Literacy: A Call to Action for Compassionate Communities
}

\author{
Lauren J. Breen \\ School of Psychology, Faculty of Health Sciences \\ Curtin University \\ Perth, Australia \\ Daisuke Kawashima \\ School of Psychology, Chukyo University, Japan
}

Karima Joy

Dalla Lana School of Public Health, University of Toronto

Toronto, ON Canada

\author{
Susan Cadell \\ Renison University College, University of Waterloo \\ Waterloo, ON Canada
}

David Roth

Pütz-Roth Bestattungen und Trauerbegleitung oHG

Bergisch Gladbach, Germany 


\begin{abstract}
Amy Chow
Department of Social Work and Social Administration, The University of Hong Kong, Hong Kong, China
\end{abstract}

Mary Ellen Macdonald

Faculty of Dentistry, McGill University

Montreal, QC Canada

\title{
Acknowledgments
}

This article was produced by the "Public Health, Health Promotion and Social Isolation" working group at the 30th meeting of the International Work Group on Death, Dying and Bereavement (IWG) held in London, Ontario, Canada, in June 2018. The IWG comprises clinicians, researchers, and educators from many countries who are dedicated to the development of research, knowledge, and practice dealing with death, dying, and bereavement.

Correspondence concerning this article should be addressed to Dr. Lauren J. Breen, School of Psychology, Curtin University, GPO Box U1987 PERTH Western Australia 6845, Australia. Email lauren.breen@curtin.edu.au 


\begin{abstract}
The compassionate communities movement challenges the notion that death and dying should be housed within clinical and institutional contexts, and works to normalize conversations about death and dying by promoting death literacy and dialogue in public spaces. Community-based practices and conversations about grief remain marginal in this agenda. We aimed to theorize how grief could be better conceptualized and operationalized within the compassionate communities movement. We develop the concept of Grief Literacy and present vignettes to illustrate a grief literate society. Grief literacy augments the concept of death literacy, thereby further enhancing the potential of the compassionate communities approach.
\end{abstract}

Key Words: Grief, death, grief literacy, compassionate communities, public health, palliative care, bereavement 


\section{Grief Literacy: A Call to Action for Compassionate Communities}

We live in communities and societies that are becoming increasingly fragmented. There are several drivers of this fragmentation, including aging populations (Myers \& Palmarini, 2017), declining fertility rates (Murray et al., 2018), mass migration and displacement (United Nations High Commissioner for Refugees, 2018), and an increase in single-person households (Snell, 2017). With increased fragmentation there is loneliness, the feeling of distress due to social isolation (Heinrich \& Gullone, 2006). Loneliness reflects the quality more than quantity of meaningful relationships (Masi, Chen, Hawkley, \& Cacioppo, 2011). The prevalence of loneliness appears to be increasing, from $11-17 \%$ in the 1970 s (Peplau, Russell, \& Heim, 1979), to over 40\% in middle aged and older adults more recently (Perissinotto, Cenzer, \& Covinsky, 2012). A recent study showed that three-quarters of a sample of community dwelling adults reported moderate to high levels of loneliness (Lee et al., 2018). Loneliness is a public health issue, the realization of which was the catalyst for the development of a Minister of Loneliness in the United Kingdom (Mead, 2016).

Loneliness is associated with many kinds of physical (Grant, Hamer, \& Steptoe, 2009) and mental health (Lim, Rodebaugh, Zyphur, \& Gleeson, 2016) issues. Chronic loneliness is equivalent to smoking 15 cigarettes per day in its association with heart disease and stroke (Valtorta, Kanaan, Gilbody, Ronzi, \& Hanratty, 2016). In contrast, strong social relationships are associated with a 50\% reduced risk of early death (Holt-Lunstad, Smith, \& Layton, 2010). If social factors make a difference in how we live and how we die, it follows then that social factors make a difference in how we grieve.

Grief is an embodied response to loss. Grief is multidimensional in that it affects all of life's domains: physical, emotional, behavioral, social, financial, and spiritual (Parkes, 1970). Historically, scholars theorized grief as an individual, intrapsychic phenomenon (Breen \& 
O'Connor, 2007; Granek, 2010), paying less attention to the impacts of wider social contexts and processes that shape the experience of grief (Breen et al., 2019; Macdonald, 2018).

Macdonald (2020) recently asserted that we live in a society that is grief-denying. The experience of grief can be compounded by the common, socially-awkward, and maladapted responses to grief. For instance, a physician reflected upon not feeling permitted to grieve a patient who died: "While I am hesitant to talk about the patient first, I am grateful that [other professionals] do, perhaps because I need an external validation that it is okay to still be grieving" (Kim, 2019, p. 1570). Bereaved people report that friends, colleagues, and health professionals "disappear," lack compassion, and offer insensitive comments and platitudes because they do not know how to be supportive (Aoun, Breen, White, Rumbold, \& Kellehear, 2018; Breen \& O’Connor, 2011; Macdonald, 2018). In 1996, in Australia's most deadly mass shooting, Walter Mikac's wife and two young daughters were killed. Over two decades later, Walter recalled that people avoided him because they did not know what to say or do, which added to his grief:

The one I think about, was my friend Doug, who I played cricket with and who was the dad of the girl who worked in my pharmacy. And one day, I was walking down the street and he was coming the other way. As soon as he saw me he turned and started walking the other way. I sort of had to make a splitsecond decision. What am I going to do? If I let him go, we'll probably never have a conversation ever again. So I started walking quicker. As I started walking quicker, he was nearly running. I caught up to him and put my hand on his shoulder and as he turned around, he just had tears streaming down his face. I said, 'It's okay, Doug, you don't have to say anything.' (Sales, 2018, pp. 55-56) 
Grief follows loss_ often, but not always, death related. Death and dying are medicalized and professionalized in many contexts (Becker et al., 2014). For example, many deaths occur following healthcare provided in hospitals, hospices, and residential aged care facilities. Following a death, bereaved people leave these institutions to return to their communities, often highly fragmented and lonely places. Further, given that much of an illness and the actual death often occurs within an institution, the community is often 'in the dark' about the illness, the death, and then the grief of those left behind.

Bereavement care is classically located in clinical and institutional forms of care (Rumbold \& Aoun, 2014). As a result, there remains a gap in social awareness of the need for bereavement care and the actual practice of providing such services for grieving people (Breen, Aoun, O’Connor, \& Rumbold, 2014; Sealey, O’Connor, Aoun, \& Breen, 2015) for a variety of reasons. One, in palliative care, bereavement care typically is only offered to those who had a connection with the service (Rumbold \& Aoun, 2014) and is minimal and sporadic (Aoun, Rumbold, Howting, Bolleter, \& Breen, 2017; Breen et al., 2014). Two, bereavement care offered within these settings tends to be provided only by mental health professionals (Granek, 2010). Three, people grieving a death that occurs outside of palliative care, and those who are too removed from the inner circle of carers, cannot easily access formalized bereavement services (Abel et al., 2013). Four, palliative care models typically start bereavement care following the death of patient rather than the beginning of the disease trajectory (Blackburn \& Dwyer, 2017). Five, grief can occur from non-death losses (e.g., divorce, family estrangement, infertility); such experiences fall far outside the purview of palliative care.

The compassionate communities movement arose in response to the increasing professionalization of healthcare, and palliative care in particular (Kellehear, 1999). The model of compassionate communities emphasizes empowering communities and harnessing 
their assets in order to contest the trend of health care being solely an institutional undertaking. A key element of the compassionate communities model is equity: that all people should have access to quality end-of-life care (e.g., regardless of age, diagnosis, or social factors). The compassionate communities movement challenges the convention that the purview of death and dying should be only within clinical and institutional contexts and instead positions it as everyone's responsibility. In so doing, this movement is working to shift the conversations about, and location of, death into community spaces.

The concept of death literacy is a relatively recent addition to this movement. Death literacy is "a set of knowledge and skills that make it possible to gain access to understand and act upon end-of-life and death care options" (Noonan et al., 2016, p. 32). The death literacy movement is gaining traction, with the establishment of massive open online courses such as Dying2Learn (Miller-Lewis, Tieman, Rawlings, Parker, \& Sanderson, 2018; Tieman, Miller-Lewis, Rawlings, \& Sanderson, 2018), Mini Death Festivals (Hilbers, Rankin-Smith, Horsfall, \& Aoun, 2018), community cemeteries (e.g., Gärten der Bestattung in Bergisch Gladbach), Shutatsu (end-of-life activities; Tanaka, Takahashi, \& Kawashima, 2019), and Death Cafes, death doulas, and FUN-erals (funerals for the living), all making contributions to the positive death movement (Leland, 2018); bereavement care is lacking in the new agenda (Aoun et al., 2018; Rumbold \& Aoun, 2014).

Recent theoretical work is addressing this challenge of bereavement care for compassionate communities. The New Essentials model (Abel, Kellehear, \& Karapliagou, 2018) more explicitly aligns with public health and community development paradigms. The model comprises specialist palliative care, generalist palliative care, compassionate communities, and civic end-of-life care, with each component including a focus on bereavement care. A recent expansion of the compassionate communities approach to develop compassionate schools, compassionate workplaces, and compassionate communities 
is taking seriously the commitment to bona fide community development that starts with the community first (Abel et al., 2013; Scottish Partnership for Palliative Care, 2018). In these community locations, not only is the dying a focus, but also, the people left behind after the death-the grievers.

In response, The Public Health Model of Bereavement Care provides a framework for community-based interventions (Aoun et al., 2015; Aoun et al., 2017; Aoun et al., 2018). This model recognizes all reactions to loss (severe reactions, less debilitating symptoms, and so-called 'normal grief'). All grievers benefit from information about grief and loss and compassionate, non-judgmental social support (Aoun et al., 2018; Penny \& Relf, 2017). A smaller proportion might benefit from grief counseling to normalize grief, assist in maintaining a connected to the deceased person, foster integration of the loss into their life story, and promote meaning and posttraumatic growth. Only a minority need specialized, psychotherapeutic interventions tailored to their needs (Aoun et al., 2015; Waller et al., 2016). The amount, timing, function, and structure of the support offered determines whether grievers perceive the offer of support to be helpful or unhelpful (Aoun, Breen, Rumbold, Christian, Same, \& Abel, 2019).

\section{Grief Literacy}

Although the Public Health Model of Bereavement Care is an important addition to the Public Health Palliative Care toolbox, it alone does not signal the paradigm shift truly required by the New Essentials (Abel et al., 2018). Instead, we believe a Grief Literacy movement is a foundational component required to move our societies forward to support bereaved people; that is, a movement to develop and expand grief literacy to benefit all people experiencing all kinds of loss.

Clark (2003) first proposed the concept of grief literacy and asserted that increasing grief knowledge would "enable the general public and professionals to identify grief more 
readily, to seek out relevant information and to adopt appropriate supports and thereby be proactive in avoiding complications from the grieving process such as depression" (p. 307). We could locate no other peer-reviewed reference to grief literacy. Building upon Clark's suggestion, we propose a definition of grief literacy as follows:

- The capacity to access, process, and use knowledge regarding the experience of loss.

- This capacity is multidimensional: it comprises knowledge to facilitate understanding and reflection, skills to enable action, and values to inspire compassion and care.

- These dimensions connect and integrate via the interdependence of individuals within socio-cultural contexts.

Our definition of grief literacy integrates the three components of knowledge, skills, and values. Their connection and integration means that grief literacy is greater than the sum of its parts. In addition, we envision that grief literacy is embedded in an inclusive, nurturing community that involves the interdependence of individuals within sociocultural contexts. Thus, grief literacy extends beyond the individual person; instead, it is a broader concept that reflects the capacity and values of a community and society. Our definition is aligned with the New Essentials (Abel et al., 2018) and provides a community-based focus, emphasizing relational as well as geographical bonds. The knowledge component includes recognizing grief, knowing how to find information, and being aware of warning signs of more complicated issues. The skills component includes listening, being able to ask questions in a sensitive manner, and being able to help individuals needing support to find resources. The values component includes ethics of care, community, and connection. Care ethics acknowledges that we live in webs of relationships with responsibilities to each other, and will all be vulnerable at some points in our lives (Tronto, 2013). Care ethics challenges dominant assumptions that we are autonomous and independent, arguing for a paradigm shift that reflects our vulnerability, relationality, and interdependence (Tronto, 2013). 


\section{Vignette Illustrations of a Grief Literate Society}

As an international group of death studies practitioners and scholars from several countries (Canada, Japan, China, Germany, and Australia) and disciplines (social work, psychology, anthropology, public health, and the funeral sector), we asked ourselves two questions: How would we know if we were grief literate? What would a grief literate society look like? These questions formed the basis of a visioning exercise wherein we determined that, in a grief literate society, people would understand and accept the uniqueness and variability of grief, rather than stigmatizing the grief of others via their own assumptions, experiences, beliefs, and expectations.

In a grief literate society, citizens would recognize and acknowledge grief from nondeath losses, and pet deaths, and not rank those losses vis-à-vis human death loss. People would understand and accept differences in grieving styles, in terms of gender (Martin \& Doka, 2000), race (Rosenblatt \& Wallace, 2014), and culture (Rosenblatt, 2008). They would feel comfortable to talk about their own loss experiences and to ask about loss experiences of others, instead of avoiding the subject or showing discomfort. Health and social care professionals would be willing to enquire about grief, would understand who needs referrals and when to refer, would have referral options available to them, and would no longer grapple with whether and how to grieve the deaths of patients and clients (Kim, 2019). Grief education would be featured in educational curricula, starting in junior levels. Further, where relevant, legislation would support the legitimacy of grief (for example, May, 2019), with attention to those in fragile economic circumstances (Corden, Hirst, \& Nice, 2010).

From this visioning exercise, we developed vignettes to capture variability in the types of loss (death and non-death), as well as who is experiencing the loss (e.g., gender, age), who died (e.g., gender, age, relationship to deceased), who/what was lost (e.g., person, pet, possession, identity, role), when the loss occurred (e.g., time since the death, caregiving 
period), where the loss occurred (e.g., place of living, surroundings), and how the loss occurred (e.g., death, displacement). The vignettes are based on our experiences as scholars and practitioners in death and grief, often portraying a desired outcome contrary to our negative personal or professional experiences. The 12 vignettes we developed begin to illustrate elements of a grief literate society.

Vignette 1 emerged from our own experiences of being hesitant to disclose publicly our work in the area of dying, death, and grief as a result of meeting discomfort when we disclose this information.

Vignette 1: Jen and Britta have just met at a barbeque. Jen asks Britta about her work and Britta says she is a counselor who works with people bereaved by suicide. Britta is confident that disclosing her work will be met with an affirming response from Jen. Jen responds, “Oh, I've never done that, tell me more."

Vignette 2 illustrates a type of loss that lacks social recognition (i.e., a miscarriage) and a griever who is less likely to receive recognition and support following a miscarriage (i.e., a father). Further, it illustrates the potential of formal bereavement leave policies and supportive workplaces. Note that Fritz's boss telephones Fritz with the offer of support, rather that Fritz having to call his boss.

Vignette 2: Fritz is preoccupied following the miscarriage of his child. Upon learning this, his boss calls and tells him, "You might not know that the government has just passed legislation for extensive bereavement leave from work. Let us know what you need right now, and when you're ready to come back to work."

Vignette 3 celebrates the power of neighbors as community assets, proactive in recognizing and responding to bereavement. In addition, it models specific actions as a 
response to grief rather than a non-committal "Let me know if..." reaction that often occurs (Soffer \& Birkner, 2017).

Vignette 3: Emiko's spouse died two weeks ago. Her neighbor comes over with a pot of soup, and, knowing it is important to check in regularly with those who are bereaved, asks: "I love to cook. Do you want to join me on Tuesdays for lunch?”

People often overlook non-death losses, which can be excluded from social assumptions about what is 'real' grief. Vignette 4 acknowledges losses that come with the refugee and immigration experience and illustrates a simple intervention by compassionate stranger.

Vignette 4: Hassan was forced to leave his dog while escaping from Syria during the civil war. Five years later, now living in Sydney, he meets Ida walking her dog. Ida notes Hassan's interest in her dog and invites him to tell his story. Ida recognizes Hassan's grief, invites him to walk the dog with her and teach her more about Syria.

Vignette 5 imagines a time when all schoolchildren know authentic histories of colonial and Indigenous nations. Note that the Indigenous speaker is hired rather than expected to share expertise for free.

Vignette 5: Phyllis hires Bill, an Indigenous speaker, to address her class by telling his people's story, focusing on resilience, intergenerational grief, and trauma. The students appreciate hearing the personal side of what is already covered in their assigned textbook.

Vignette 6 considers the grief of a child experiencing the death of a sibling. It celebrates the ability of other children to recognize warning signs of something potentially harmful occurring and to know who to turn to for help. 
Vignette 6: Carlos is 12 years old. With the anniversary of his twin brother's death approaching, his football teammates are more attentive to Carlos. They notice that, despite the heat, Carlos will only wear long-sleeved jerseys. Worried that Carlos might be self-harming, the teammates approach the coach for advice.

Vignette 7 acknowledges that grief can be particularly difficult in the context of a young age, queer culture, and that contagion can occur in communities. The response illustrates how governments should be able to respond.

Vignette 7: In the aftermath of a string of youth suicides in a rural community, the federal government moves quickly to respond. They take an upstream approach and increase funding to agencies with a mandate of suicide prevention for all ages. In addition, with the recognition that LGBTQ+ youth experience higher rates of suicide, the government releases funds to community groups that support them with a requirement that part of the funds go to community-based initiatives, including public service announcements. Vignette 8 celebrates public education about grief as well as underlining the utility of advance care plans and directives.

Vignette 8: After attending an information session at the public library about grief and trauma, Rashid reflects on the death of his partner Ben, who died suddenly of a heart attack, seven years earlier. Rashid is grateful that they had had numerous conversations about advance directives, funeral planning, and future grief. He attributes his lack of traumatic response, despite the unexpected death, to the preparation that the two of them had.

Vignette 9 suggests that, when accidents and disasters occur, communities can respond collectively. 
Vignette 9: A school community is rocked by the news that three students from one family have died in a house fire over the summer break. When the fall session begins, the school board brings in numerous resources for the children, parents, and staff to support their grief in the weeks that follow. A committee of school personnel, parents, and students later hold a memorial service at the school for anyone wishing to attend. Vignette 10 celebrates how together, palliative care practitioners and legislation can facilitate caregiving and bereavement experiences.

Vignette 10: Karen was on caregiver leave until her partner died. The palliative care workers facilitated access to resources and community supports throughout the illness and after the death. In addition, Karen is grateful for an extended caregiver bereavement leave from work which protects her job and provides a salary, so she does not feel pressure to return to work prematurely.

Vignette 11 recognizes that the death of a child can be extremely difficult for parents. It uplifts supportive presence and even silence as a possible supportive tool.

Vignette 11: John learns from the neighborhood association that his neighbors' child has died. John had previously attended an information session by the association about grief. John chooses to go over to his neighbors' house to offer a supportive presence. He does not try to fill the silence, knowing there is nothing he can say to take the pain away and that he is being supportive by showing them they are not alone.

Vignette 12 demonstrates how workplaces can implement policies that improve the lives of those who are grieving.

Vignette 12: With knowledge that grief does not follow a linear timeline, Clara's workplace has initiated a flexible bereavement leave policy so that employees can 
take leave when they need it. The workplace does not stigmatize or punish employees for taking time off.

\section{Call to Action}

We sought to illustrate components of an imagined grief literate society via these vignettes. Workplaces and governments can implement policies that provide flexibility and protections to workers (Macdonald et al., 2015). For example, in the United Kingdom, The Parental Bereavement law (Leave and Pay) means that, starting April 2020, bereaved parents will be entitled to two weeks' paid leave after the death of a child. Canada has just gone through a federal process to consider extending supports for parental bereavement as well (Macdonald, 2020). There is also a campaign to extend bereavement leave in the Australian National Employment Standards (https://memoleaves.com/bereavement-leave/).

Our vision of grief literacy inspires this call to action. A paradigm shift is necessary to develop grief literate cultures and societies, including context-specific explorations of opportunities and barriers to change. These shifts will take time and effort; grief literacy will not happen overnight. For example, literature shows that having personal experience of bereavement is not sufficient in determining the likelihood of offering grief support following a death (Logan, Thornton, Kane, \& Breen, 2018). Grief resources online, such as Modern Loss (modernloss.org) and My Grief (mygrief.ca) seek to educate people about grief, to differentiate it from other experiences (e.g., trauma, depression), and to enable people to respond to and support those who are grieving. A grief literate society will only occur as an outcome of education and action: through health promotion and community development. We suggest three starting points, as follows.

First, communities should carefully consider how to promote grief literacy locally. Communities need to determine for themselves the knowledge required, the skills needed to enact it, and the values relevant to their cultural context. These aspects will differ depending 
upon ethnicity, culture, gender, sexual orientation, age, religion, abilities, and other socioeconomic factors. At its heart, achieving grief literacy is an educational undertaking. Positive educational approaches must build on existing knowledge, which may include expanding and challenging assumptions. Communities should determine the content of this education and make access to it available to all. The vignettes we developed may be one tool to achieve this.

Second, researchers, health professionals, and practitioners need to work alongside communities to develop and evaluate grief literacy initiatives. Initiatives should take an upstream approach to promoting collective grief literacy rather than only responding to individual-level acute grief (as we emphasize in the vignettes). In so doing, they must help identify those with higher needs who hesitate to seek support (Lichtenthal et al., 2011). Thus, we highlight a dual focus: the development and availability of specialized services while also investing in building the community's capacity to understand grief and provide supportive, compassionate care. These initiatives should be as well-developed as death literacy initiatives. Although there are long-standing markers of culturally-sanctioned grief (e.g., Easter in Christian communities, Tomb Sweeping Day in China, Bon Period in Japan, Remembrance Day in Canada, ANZAC Day in Australia and New Zealand), none of these aims to build community capacity in grief. Some grassroots, community-based initiatives already aim to promote public grieving, and in so doing, perhaps increase grief literacy. Some examples are Blue Christmas (Canada), Sorry Business (Australia), To Absent Friends (Scotland), Buddy Group (UK; Riley et al., 2018), and The Compassionate Friends (international). Grassroots groups and other peer support/befriender models for grief have overt advocacy and community education mandates. Understanding how these initiatives work, and how they could be harnessed, tailored, modeled, and scaled up for broader public education is essential. 
Third, we must be wary of an unintended consequence that may arise from positioning bereavement care as a community responsibility. Importantly, existing services could refrain from providing bereavement care if they view the community as the sole provider of such support. Austerity measures associated with neoliberal values drive socially irresponsible and premature funding cuts, prioritizing costs over wellbeing (Tronto, 2013). We require adequate systems and authentic community partnerships to ensure the development of grief literacy is integrated with health and social care professionals already providing direct support. The community counseling model provides an exemplar for service delivery that combines direct and indirect services to individuals and communities (Lewis et al., 2003). In this model, direct services aimed at the community involve interventions that reduce the need for professional one-on-one assistance, such as educational seminars and workshops. Direct client services include providing individualized, therapeutic, and professional interventions aimed at people in need and at-risk, including specialist psychotherapy. Indirect community services involve activities such as influencing public policy in order to support the community and creating systemic change. Indirect client services involve client advocacy and consultation, such as educating the wider community and supporting the development of mutual-help groups. The model offers a unified approach to assist services to develop a multi-faceted and complementary combination of programs and interventions in partnership with their communities.

Grief literacy is not an intervention, per se; rather, it is a paradigm shift that addresses explicitly the social contexts influencing how we grieve. In presenting vignettes of grief literacy, we seek to illustrate what can be accomplished through education and action at micro, meso, and macro-levels. We anticipate the collateral benefits of grief literacy will include increases in collective wellbeing, resilience, community cohesion, social fabric, and emotional competence, and reductions in loneliness, isolation, struggle, stigma, and suicide. 
Future research should develop and assess the effectiveness of interventions that promote grief literacy and examine context-specific drivers and barriers of change. Grief literacy must complement death literacy in order to realize the full potential of compassionate communities, because a grief literate society necessarily comprises people who are death literate, too. 


\section{References}

Abel, J., Kellehear, A., \& Karapliagou, A. (2018). Palliative care-the new essentials. Annals of Palliative Medicine, 7, S3-S14. doi:10.21037/apm.2018.03.04

Abel, J., Walter, T., Carey, L. B., Rosenberg, J., Noonan, K., Horsfall, D., ... \& Morris, D. (2013). Circles of care: Should community development redefine the practice of palliative care? BMJ Supportive \& Palliative Care, 3, 383-388. doi:10.1136/bmjspcare-2012-000359

Aoun, S. M., Breen, L. J., Howting, D. A., Rumbold, B., McNamara, B., \& Hegney, D. (2015). Who needs bereavement support? A population based survey of bereavement risk and support need. PloS One, 10(3), e0121101. doi:10.1371/journal.pone.0121101

Aoun, S. M., Breen, L. J., Rumbold, B., Christian, K. M., Same, A., \& Abel, J. (2019). Matching response to need: What makes social networks fit for providing bereavement support? PLoS ONE 14(3): e0213367. https://doi.org/10.1371/journal.pone.0213367

Aoun, S. M., Breen, L. J., White, I., Rumbold, B., \& Kellehear, A. (2018). What sources of bereavement support do bereaved people perceive to be helpful and why? Empirical evidence for the compassionate communities approach. Palliative Medicine, 32, 13781388. doi:10.1177/0269216318774995

Aoun, S. M., Rumbold, B., Howting, D. A., Bolleter, A., \& Breen, L. J. (2017). Bereavement support for family caregivers: The gap between guidelines and practice in palliative care. PloS One, 12(10):e0184750. doi:10.1371/journal.pone.0184750

Becker, C., Clark, E., DeSpelder, L. A., Dawes, J., Ellershaw, J., Howarth, G., . . \& the International Work Group on Death, Dying and Bereavement. (2014). A call to action: An IWG charter for a public health approach to dying, death, and loss. Omega: Journal of Death and Dying, 69, 401-420. doi:10.2190/OM.69.4.d 
Blackburn, P., \& Dwyer, K. (2017). A bereavement common assessment framework in palliative care: Informing practice, transforming care. American Journal of Hospice and Palliative Medicine, 34, 677-684. doi:10.1177/1049909116647403

Breen, L. J., Aoun, S. M., O’Connor, M., \& Rumbold, B. (2014). Bridging the gap in palliative care bereavement support: An international perspective. Death Studies, 38, 54-61. doi:10.1080/07481187.2012.725451.

Breen, L. J., \& O’Connor, M. (2007). The fundamental paradox in the grief literature: A critical reflection. Omega: Journal of Death and Dying, 55, 199-218. doi:10.2190/OM.55.3.c

Breen, L. J., \& O'Connor, M. (2011). Family and social networks after bereavement: Experiences of support, change, and isolation. Journal of Family Therapy, 33, 98-120.

Breen, L. J., Szylit, R., Gilbert, K. R., Macpherson, C., Murphy, I., Nadeau, J. W., Reis e Silva, D., Wiegand, D. L., \& the International Work Group on Death, Dying, and Bereavement. (2019). Invitation to grief in the family context. Death Studies, 43, 173182. doi:10.1111/j.1467-6427.2010.00495.x

Clark, S. E. (2003). Loss and grief in general practice: The development and evaluation of two instruments to detect and measure grief in general practice patients. Unpublished thesis, University of Adelaide, Australia. Available from https://digital.library.adelaide.edu.au/dspace/handle/2440/37929

Corden, A., Hirst, M., \& Nice, K. (2010). Death of a partner: Financial implications and experience of loss. Bereavement Care, 29(1), 23-28.

Granek, L. (2010). Grief as pathology: The evolution of grief theory in psychology from Freud to the present. History of Psychology, 13, 46-73. doi:10.1037/a0016991 
Grant, N., Hamer, M., \& Steptoe, A. (2009). Social isolation and stress-related cardiovascular, lipid, and cortisol responses. Annals of Behavioral Medicine, 37, 2937. doi:10.1007/s12160-009-9081-z

Heinrich, L. M., \& Gullone, E. (2006). The clinical significance of loneliness: A literature review. Clinical Psychology Review, 26, 695-718. doi:10.1016/j.cpr.2006.04.002

Hilbers, J., Rankin-Smith, H., Horsfall, D., \& Aoun, S. (2018). "We are all in this together": Building capacity for a community-centred approach to caring, dying and grieving in Australia. European Journal for Person Centered Healthcare, 6, 685-692.

Holt-Lunstad, J., Smith, T. B., \& Layton, J. B. (2010). Social relationships and mortality risk: A meta-analytic review. PloS Medicine, 7(7): e1000316. doi:10.1371/journal.pmed.1000316

Kellehear, A. (1999). Health promoting palliative care. Oxford, UK: Oxford University Press.

Kim, M. (2019). The right to grieve. JAMA, 321(16), 1569-1570. doi:10.1001/jama.2019.4265

Lee, E. E., Depp, C., Palmer, B. W., Glorioso, D., Daly, R., Liu, J., .. \& Jeste, D. V. (2018). High prevalence and adverse health effects of loneliness in community-dwelling adults across the lifespan: Role of wisdom as a protective factor. International Psychogeriatrics. doi:10.1017/S1041610218002120

Leland, J. (2018, June 22). The positive death movement comes to life. The New York Times. Lewis, J. A., Lewis, M. D., Daniels, J. A., \& D’Andrea, M. J. (2003). Community counseling: Empowerment strategies for a diverse society ( $3^{\text {rd }}$ ed.). Pacific Grove, CA: Thomson. Lichtenthal, W. G., Nilsson, M., Kissane, D. W., Breitbart, W., Kacel, E., Jones, E. C., \& Prigerson, H. G. (2011). Underutilization of mental health services among bereaved 
caregivers with prolonged grief disorder. Psychiatric Services, 62, 1225-1229. doi:10.1176/appi.ps.62.10.1225

Lim, M. H., Rodebaugh, T. L., Zyphur, M. J., \& Gleeson, J. F. M. (2016). Loneliness over time: The crucial role of social anxiety. Journal of Abnormal Psychology, 125, 620630. doi:10.1037/abn0000162

Logan, E. L., Thornton, J. A., Kane, R. T., \& Breen L. J. (2018). Social support following bereavement: The role of beliefs, expectations, and support intentions. Death Studies, 42, 471-482. doi:10.1080/07481187.2017.1382610

Macdonald, M. E. (2018, February 6). Why should the bereaved give up their ghosts? Discover Society.

Macdonald, M. E. (2020). The denial of grief: Reflections from a decade of anthropological research on parental bereavement and child death. In M. H. Jacobsen \& A. Petersen (Eds.), Exploring grief: Towards a sociology of sorrow (pp. 125-149): London: Routledge.

Macdonald, M. E., Kennedy, K., Molls, S., Pineda, C., Mitchell, L. M., Stephenson, P. H., \& Cadell, S. (2015). Excluding parental grief: A critical discourse analysis of bereavement accommodation in Canadian labour standards. Work: A Journal of Prevention, Assessment and Rehabilitation, 50, 511-526.

Martin, T. L., \& Doka, K. J. (2000). Men don't cry... women do: Transcending gender stereotypes of grief. Philadelphia: Brunner/Mazel.

Masi, C. M., Chen, H. Y., Hawkley, L. C., \& Cacioppo, J. T. (2011). A meta-analysis of interventions to reduce loneliness. Personality and Social Psychology Review, 15, 219-266. doi:10.1177/1088868310377394 
May, B. (2019). Supporting Families after the Loss of a Child. In Report of the Standing Committee on Human Resources, Skills and Social Development and the Status of Persons with Disabilities. Ottawa, ON: Government of Canada.

Mead, R. (2016, January 26). What Britain's “Minister of Loneliness” says about Brexit and the legacy of Jo Cox. The New Yorker.

Miller-Lewis, L., Tieman, J., Rawlings, D., Parker, D., \& Sanderson, C. (2018). Can exposure to online conversations about death and dying influence death competence? An exploratory study within an Australian massive open online course. Omega: Journal of Death and Dying, advance online publication. doi:10.1177/0030222818765813

Murray, C. J. L., Callender, C. S. K. H., Kulikoff, X. R., Srinivasan, V., Abate, D, Abate, K., H., ... \& Lim, S. S. (2018). Population and fertility by age and sex for 195 countries and territories, 1950-2017: A systematic analysis for the Global Burden of Disease Study 2017. The Lancet, 392, 1995-2051. doi:10.1016/S0140-6736(18)32278-5

Myers, L., \& Palmarini, N. (2017). Loneliness: Findings from IBM's Institute for Business Value 2017 Study on Loneliness in the Aging Population. Public Policy and Aging Report, 27(4), 158-159. doi:10.1093/ppar/prx022

Noonan, K., Horsfall, D., Leonard, R., \& Rosenberg, J. (2016). Developing death literacy. Progress in Palliative Care, 24(1), 31-35. doi:10.1080/09699260.2015.1103498

Parkes, C. M. (1970). 'Seeking' and 'finding' a lost object: Evidence from recent studies of the reaction to bereavement. Social Science and Medicine, 4, 187-201.

Penny, A., \& Relf, M. (2017). A guide to commissioning bereavement services in England. National Bereavement Alliance. Accessed June 272018 from www.nationalbereavementalliance.org.uk 
Peplau, L. A., Russell, D., \& Heim, M. (1979). The experience of loneliness. In I. H. Frieze, D. Bar-Tal, \& J. S. Carroll (Eds.), New approaches to social problems: Applications of attribution theory (pp. 53-78). San Francisco, CA: Jossey-Bass.

Perissinotto, C. M., Cenzer, I. S., \& Covinsky, K. E. (2012). Loneliness in older persons: A predictor of functional decline and death. Archives of Internal Medicine, 172, 10781083. doi:10.1001/archinternmed.2012.1993.

Riley, S. G., Pettus, K. I., Abel, J. (2018). The buddy group - peer support for the bereaved. London Journal of Primary Care, 10, 68-70. doi:10.1080/17571472.2018.1455021

Rosenblatt, P. C. (2008). Grief across cultures: A review and research agenda. In M. Stroebe, R. O. Hansson, H. Schut, \& W. Stroebe (Eds.), Handbook of bereavement research and practice: Advances in theory and intervention (pp. 207-222). Washington, DC: American Psychological Association.

Rosenblatt, P. C., \& Wallace, B. R. (2013). African American grief. New York, NY. Routledge.

Rumbold, B., \& Aoun, S. (2014). Bereavement and palliative care: A public health perspective. Progress in Palliative Care, 22, 131-135. doi:10.1179/1743291X13Y.0000000079

Sales, L. (2018). Any ordinary day: Blindsides, resilience, and what happens after the worst day of your life. Melbourne, Australia: Penguin Books.

Scottish Partnership for Palliative Care. (2018). A road less lonely: Moving forward with public health approaches to death, dying and bereavement in Scotland. Author. Accessed June 272018 from https://www.palliativecarescotland.org.uk/

Sealey, M., O’Connor, M., Aoun, S. M., \& Breen, L. J (2015). Exploring barriers to assessment of bereavement risk in palliative care: Perspectives of key stakeholders. BMC Palliative Care, 14:49. doi:10.1186/s12904-015-0046-7. 
Snell, K. D. M. (2017). The rise of living alone and loneliness in history. Social History, 42, 2-28. doi:10.1080/03071022.2017.1256093

Soffer, R., \& Birkner, G., (2017). Modern loss: Candida conversations about grief: Beginners welcome. New York, NY: Harper Wave.

Tanaka, M., Takahashi, M., \& Kawashima, D. (2019). End-of-life activities among community-dwelling older adults in Japan. Omega: Journal of Death and Dying, advance online publication. doi:10.1177/0030222819854926

Tieman, J., Miller-Lewis, L., Rawlings, D., \& Sanderson, C. (2018). The contribution of a MOOC to community discussions around death and dying. BMC Palliative Care, 17, 31. doi:10.1186/s12904-018-0287-3

Tronto, J. (2013). Caring democracy: Markets, equality, and justice. New York: NYU Press. United Nations High Commissioner for Refugees. (2018). Global trends: Forced displacement in 2017. Accessed 8 May 2019 from https://www.unhcr.org/globaltrends2017/\#

Valtorta, N. K., Kanaan, M., Gilbody, S., Ronzi, S., \& Hanratty, B. (2016). Loneliness and social isolation as risk factors for coronary heart disease and stroke: Systematic review and meta-analysis of longitudinal observational studies. Heart, 102, 10091016. doi:10.1136/heartjnl-2015-308790

Waller, A., Turon, H., Mansfield, E., Clark, K., Hobden, B., \& Sanson-Fisher, R. (2016). Assisting the bereaved: A systematic review of the evidence for grief counselling. Palliative Medicine, 30, 132-148. doi:10.1177/0269216315588728 